\title{
Investigation of Interference Effects for a Group of Finite Cylinders
}

\author{
A. Kareem ${ }^{1}$, Tracy Kijewski ${ }^{1}$, Po-Chien Lu $^{2}$ \\ 1. University of Notre Dame, NatHaz Modeling Laboratory, Dept. of Civil Engineering and Geological Sciences \\ 2.Tamkang University, Taipei, Taiwan
}

\begin{abstract}
An experimental study of the interference effects between two and three cylinders of finite height immersed in a turbulent boundary layer at subcritical Reynolds numbers has been conducted in a boundary layer wind tunnel, utilizing a pneumatic averaging manifold system to measure the fluctuating force at various levels. Measurements of mean drag and lift force coefficients, mode-generalized RMS drag and lift force coefficients, and mode-generalized drag and lift force spectra, for a range of cylinder spacings and various angles of attack, are presented. As one may expect, the interference levels observed depend strongly on the spacing, angle of attack, and the arrangement of the cylinders with respect to each other. This study concerning the investigation of these relationships for finite cylinders should prove insightful to those interested in such phenomena.
\end{abstract}

\section{Introduction}

Flow behavior around circular cylinders is a classical problem in fluid mechanics with a variety of practical applications, ranging from tall chimneys exposed to atmospheric boundary layer flows to cooling systems of nuclear reactors. The proximity of the adjacent structures under certain conditions introduces adverse or beneficial effects. From an aerodynamics perspective, a strong interaction takes place in the flow field around multiple body configurations that are sensitive to approach flow characteristics, as well as the angle of attack.

A host of studies have addressed the interference effects between two, three, and even four cylinders of finite height in uniform and/or turbulent flow, ${ }^{1-23}$ which has been the focus of efforts in recent decades. Still, there is much less information on the aerodynamic characteristics of multiple finite cylinders. In what experimental work that has been done, primary focus has been devoted to the influence of aspect ratio and cylinder spacing on aerodynamic behavior in subcritical, low-turbulence flows. The majority of this work has focused on the calculation of the mean and fluctuating pressure distributions on cylinders in tandem as described Luo, et. al. ${ }^{9}$ Some attention has also been given to other configurations such as staggered and side-by-side alignments in work by Sun and $\mathrm{Gu}^{10}$ and Sun, et. al. ${ }^{11}$ Still, the majority of work has been dedicated to the study of localized effects, with few studies giving primary consideration to lift and drag forces, especially their fluctuating components 5 and their spectra. Furthermore, since most of the previous work was carried out in smooth flow, there is a shortage of information regarding the fluctuating forces and pressures on cylinders in turbulent boundary layer flows.

Besides fundamental differences in the shear layer approach flow around cylinders in comparison with uniform flow, an additional feature is introduced in finite height cylinders: the flow over the cylinder's top face ${ }^{12}$. At the top, the shear layer separates and interacts with the two layers which separate form the sides of the 
cylinder. ${ }^{9}$ The present study considers this situation for a group of two and three cylinders of finite height and equal diameter, tested in a simulated boundary layer to investigate the aerodynamically-induced fluctuating forces, as would be experienced by a group of chimney stacks. In particular, the present study details the relationship between cylinder spacing and arrangement (e.g. tandem, side-by-side, or staggered) and the level of interference which results. The level of interference between the cylinders will be reflected by the mean drag and lift coefficients, mode generalized RMS drag and lift coefficients, and the mode generalized drag and lift force spectra. All measurements are restricted to open country type flow, as the interference effects due to adjacent bodies are most pronounced in flows with low turbulence levels.

\section{Experimental Apparatus and Method}

The experiments were conducted in a boundary layer wind tunnel featuring a test section $4 \mathrm{ft}$. 7 in. high, $9 \mathrm{ft} .10$ in. wide, and $47 \mathrm{ft}$. long. The atmospheric boundary layer was simulated by passing the natural wind over surface roughness along the tunnel floor and past spires and barriers at the entrance to the testing section. A boundary layer 40 in. thick with a power law exponent of 0.16 to model an open country condition at a Reynolds number of $2.7 \times 10^{4}$ was used in the study.

A 3 in. x 30 in. circular plexiglass cylindrical model, instrumented with pressure taps, was used for the wind load measurement. The dummy cylinders, serving as the adjacent interfering cylinders, were also constructed of plexiglass but without pressure taps. The model's height was divided into five levels, each with 14 pressure taps, placed such that groups of seven taps were located on each semi-circle of the cylinder, so that the tributary arc length for each tap resulted in an identical value of $\sin (\theta) d \theta$, in which $\theta$ is the angle between the line joining the tap location and the origin with the horizontal axis, and $d \theta$ is the arc angle subtended by the tributary arc length. Each of the seven pressure tap groups was connected to a seven-input manifold. The two manifolds facing one another on each level were connected to a pressure transducer to yield aerodynamic loads at that level. In this study, the levels along the height, at which local forces were measured by a manifolding technique, were spaced such that the summation of forces at that level provided appropriate weighting, resulting in a mode-generalized force. The pressure tubing and pneumatic averaging manifold were dynamically calibrated and their transfer function was directly incorporated in the signal processing to obtain high frequency response for the system. The simultaneously-measured output signals were passed through a low-pass filter, which were then digitized for subsequent analysis.

The mean drag and lift force coefficients were obtained through a summation of the five levels of pneumatically averaged area loads and then normalized with a factor of $1 / 2 \rho U_{H}^{2} D H$, where $\rho$ is the density of the air, $U_{H}$ is the velocity of the boundary layer at the height of the cylinder, and $D$ and $H$ are the diameter and height of the cylinder, respectively.

The generalized spectra were obtained by covariance integration which requires knowledge of the spanwise correlation and takes mode shape into account. The generalized RMS force was obtained by taking the square root of the generalized spectra, which was then normalized by a factor of $1 / 2 \rho U_{H}^{2} D H$, as defined above.

\section{Test Configurations}

As outlined in Fig. 1, measurements were taken at $\theta=0^{\circ}, 10^{\circ}, 20^{\circ}, 30^{\circ}, 40^{\circ}$, and $90^{\circ}$ at spacings of $\mathrm{S} / \mathrm{D}=$ 2, 3, 4, 5,6 and 7 for a set of two cylinders labeled configuration 1, where $S$ is the distance between the centers of the cylinders and D is the diameter of the cylinders. For the case of three cylinders, a similar configuration (Fig. 1, configuration 2) was observed but for spacings only up to 5. In these scenarios, the observed cylinder was one of three identical cylinders placed in line and located either at the middle or ends, with the spacing measured 
center to center symmetrically about the middle cylinder. A third configuration, three cylinders is in a staggered arrangement, was also studied for two cases: cylinder A in either a windward or leeward position, also shown in Fig. 1. The cylinder under observation was positioned in the center line of the two side-by-side dummy cylinders, with logitudinal separations of $\mathrm{L} / \mathrm{D}=-3,-2,2,3,4$ and 5 . The negative sign and positive sign correspond to the measurement cylinder being upstream and downstream of the two dummy cylinders, respectively. The two dummy cylinders were separated transversely at spacings of $\mathrm{T} / \mathrm{D}=2,3$ and 4 . For all cases, the behavior of an isolated cylinder is presented for comparison. In the discussions which follow, although measurements were made for the incident angles of $\theta$ mentioned above, only results for $\theta=0^{\circ}$ (tandem) and $90^{\circ}$ (side by side) will be discussed in detail herein, with passing comments on the other intermediate orientations. In addition, while spectra and plots of the variation of the force coefficients with spacing were generated for all configurations studied, for brevity's sake, not all could be presented in this paper and are just commented on in passing.

\section{Results and Discussion}

\section{Configuration 1: Two Cylinders}

Tandem Arrangement: In the tandem configuration, the cylinders are one behind the other in the oncoming flow, creating a situation where the downstream bodies are enveloped in the wake of the upstream cylinder. In such instances, one would expect the interference of the flow between the cylinders to be closely connected to the properties and behavior (e.g. shear layers, vortex formation, etc.) of the upstream wake ${ }^{14}$, with the mean drag forces decrease remarkably at small spacings and for small angles ${ }^{8,15}$. The mean drag force of the downstream cylinder is strongly affected by the upstream wake (Fig. 2a). Once in the tandem configuration, the downstream cylinder experiences a lower drag force for almost all spacings. This phenomena results from the UC spawning a turbulence structure that promotes attachment of flow onto the downstream cylinder creating a change in pressure distribution which translates into a rapid decrease in the drag force. However, the minimum

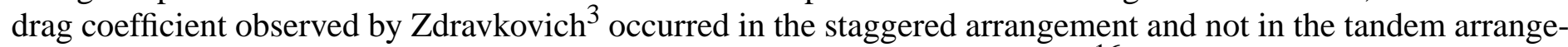
ment for two 2D cylinders in a uniform flow. Similarly, a study by Sun and $\mathrm{Gu}^{16}$ observed a minimum drag force between $5^{\circ}$ and $10^{\circ}$, which, although staggered, is close to the tandem configuration. The mean drag force $C_{D}$ acting on the upstream cylinder (UC) is slightly subject to the influence of the downstream cylinder (DC) only when the spacing between them is less than 4 cylinder diameters, in agreement with the findings of Taniguchi, et al. ${ }^{15}$ For spacings less than this critical spacing, $C_{D}$ is lower than that of isolated cylinder.

The mean lift force is almost zero for the tandem arrangement due to the symmetrical side force on the cylinder, while the maximum negative lift force occurs near in the staggered configuration for $\theta=10^{\circ}$. The negative lift force increases as the downstream cylinder approaches the upstream wake at small separations. This trend observed in this study shows agreement with that of Bokian and Geoola ${ }^{8}$, Taniguchi, Sakamoto and Arie ${ }^{15}$, and Zdravkovich and Pridden ${ }^{3}$. Ishigiai, et al. ${ }^{17}$ also observed this trend for a tandem arrangement with critical spacing being 3.8 diameters, noting the development of vortex streets behind both cylinders equal values of vortex shedding frequency. The behavior of the flow in the tandem arrangement may be classified into two regimes: For spacings up to the critical spacing, the vortex street is suppressed behind the front cylinder and beyond this critical spacing, both cylinders form vortex streets. ${ }^{14}$

The generalized force coefficient represents the local drag or lift force weighted with the fundamental modes. The buffeting effect of the wake on the DC in general shows an increase in the fluctuating drag irrespective of the spacing. The generalized drag of the UC is insignificantly affected by the presence of the downstream cylinder and only shows a small reduction at small spacings. However, the generalized RMS lift force coefficient $\left(\tilde{C}_{L}\right)$ is reduced for spacing ratios less than 3, as illustrated in Fig. 2b. The reductions of these fluctuating forces are a result of the vortex shedding from the upstream cylinder being suppressed at small separations due to the 
obstruction introduced by the downstream cylinder.

An exceptional feature surfaces for the DC in the tandem arrangement: both the RMS drag and lift forces at $\mathrm{S} / \mathrm{D}=2$ are larger in comparison to the isolated cylinder, while, for $\mathrm{S} / \mathrm{D}=3$, they converge to a narrower range of values produced by other angles. Results here are plotted in Fig. 2b for RMS lift only. A similar observation was also made by Arie, et al. ${ }^{5}$ It is not surprising that the interference is very high for both drag and lift force fluctuations. For all configurations except side-by-side $\left(\theta=90^{\circ}\right)$, the RMS drag reaches a maximum at $S / D=4$ and then slowly decays, in agreement with other studies ${ }^{3,5}$. When $\theta=20^{\circ}$, the downstream cylinder experiences the highest RMS drag between $\mathrm{S} / \mathrm{D}=3$ to 6 . As $\theta$ increases beyond $10^{\circ}$, the RMS lift is not much affected by the presence of the upstream cylinder; however, the interference is considerably high for angles of $0^{\circ}$ and $10^{\circ}$. For all angles from $S / D=2$ to 4 , there is a rapid increase in RMS lift, except for $\theta=90^{\circ}$. At $S / D=4$, the RMS lift for angles of $30^{\circ}$ and $40^{\circ}$ falls rapidly, but those of $0^{\circ}$ and $10^{\circ}$ remain constant even for spacing $\mathrm{S} / \mathrm{D} \geq 7$. The maximum RMS lift occurs along $\theta=20^{\circ}$ for spacing $S / D=3$ to 6 . It is noted that both the RMS drag and RMS lift peak for flow approaching at $\theta=20^{\circ}$. In this configuration, the shear layer of the UC has maximum impact on the DC forces.

The preceeding observations concerning the generalized force coefficients are reflected in their corresponding spectral descriptions. Though not shown here, suppressed vortex shedding at S/D $\leq 3$ is characterized by the broader peak in the generalized spectra in the acrosswind direction for the UC, although the spectra for the alongwind force look very much the same as that of isolated cylinder for all $\theta$ and S/D. For S/D > 3, vortices begin to shed periodically from the upstream cylinder, and interference will commence in the fully turbulent wake region. For these larger separations $(\mathrm{S} / \mathrm{D}>2)$, the spectra show a shape similar to that of an isolated cylinder, featuring a sharp peak.

While the DC shows similar trends, the acrosswind spectrum of the downstream cylinder, shown in Fig. 3c, exhibits a peak which is slightly higher and exceptionally narrow compared to the isolated cylinder (Fig. 3a), indicating a particularly strong periodicity and coherence of the vortex shedding along the cylinder height. At S/ $\mathrm{D} \leq 3$, the sharp peak is suppressed and diminishes in magnitude with a broader band. At $\mathrm{S} / \mathrm{D}=3$ with $\theta=10^{\circ}$ and $20^{\circ}$, the peak does not appear.

On the other hand, the alongwind spectrum of the downstream cylinder at the tandem arrangement $(\theta=$ $0^{\circ}$ ) has a pronounced peak in the vicinity of the double the shedding frequency or so called longitudinal mode. Upon approach flow angle change, the downstream cylinder facilitates a gap flow which is biased towards the DC. The axes of the vortex shedding of the downstream cylinder skewed away from the centerline of the wake; therefore, the alongwind spectra of the downstream cylinder shows a fundamental peak at a frequency corresponding to the vortex shedding coupled with a second harmonic peak at twice of the shedding frequency. This second peak is also discernible for $\mathrm{S} / \mathrm{D}>3$ when $\theta=10^{\circ}$ (as shown in Fig. 3(d)) and $20^{\circ}$. The first peak diminishes in magnitude with increasing $\mathrm{S} / \mathrm{D}$ and $\theta$, becoming almost indiscernible for $\mathrm{S} / \mathrm{D}>5$ and $\theta=30^{\circ}$ and $\mathrm{S} / \mathrm{D}>$ 4 with $\theta=40^{\circ}$. Depending on the angle of attack, the wake of the UC begins to interact with the flow over the DC, thus modifying its wake. With increasing angle of the approach flow, additional harmonics in the spectra begin to appear.

Side by Side Configuration: In the side by side arrangement, it has been observed that for sufficiently wide spacings (typically beyond 3.5 S/D), the two cylinders will form their own vortex streets independently, like that of an isolated cylinder ${ }^{14}$; however, at diminished spacings, antiphase vortex shedding appears (the production of vortices of opposite sign by each cylinder, simultaneously). While this symmetry is maintained for spacings at a spacing of 2 , even closer spacings result in a biased flow biased to one side with a large scale Karman 
vortex street formed some distance downstream. At very small spacings, the gap flow is weak and separated shear layers on the outside of both cylinders interact with each other and roll up to form a large-scale vortex, thus yielding the behavior of an isolated cylinder. Such bistable behavior will also be reflected in the drag and lift coefficients. The cylinder on the biased side will experience larger drag and lift forces that are associated with a narrower wake, the converse being true for the cylinder on the unbiased side. It has also been shown that the sum of the bistable high and low drag is always less than twice the drag of a single cylinder ${ }^{14}$. This biased flow phenomena was also observed in side by side arrangements at supercritical Reynolds numbers in a study by Sun and $\mathrm{Gu}^{16}$. On the other hand, cylinders in the side by side configuration have been observed to have the biased side cylinder experience a larger drag and lift force associated with a narrow wake, while the other cylinder on the unbiased side has smaller drag and lift forces associated with a wide wake ${ }^{14}$.

In support of previous observations, the interference between the cylinders becomes negligible for $S / D \geq$ 2. The variations and the values of the RMS lift of the downstream cylinder are considerably higher than those of the RMS drag, though the RMS lift exhibits a lower peak at $S / D=3$. A further understanding of the RMS phenomena, though not shown here in a figure, can be gained from the corresponding spectra which reveal that the amplitude of peaks at the frequency corresponding to vortex shedding and a second harmonic peak at twice the shedding frequency are sensitive to the spacings resulting in a redistribution of spectral energy in the side by side configuration.

\section{Three Cylinders Case}

\section{Configuration 2: Three Cylinders}

It may be noted that the interference acting on the middle cylinder (MC) are much the same as those of the downstream cylinder in the two cylinders case. The mean drag and the mean lift of the end or downstream cylinder in the three cylinders case exhibits only a slight difference from values for the middle cylinder. However, the RMS drag coefficient of the third cylinder is greater than that of the second one, especially for $\theta=30^{\circ}$, in which case the value of the RMS drag coefficient becomes comparable with that for $\theta=20^{\circ}$. The variation is more marked for RMS lift coefficient. At $\theta=30^{\circ}$, the RMS lift coefficient increases rapidly and reaches a level as high as that for $\theta=20^{\circ}$ at $\mathrm{S} / \mathrm{D}=4$, then decreases rapidly and backs to a level as low as those for $0^{\circ}$ and $10^{\circ}$. The RMS lift coefficients for $0^{\circ}$ and $10^{\circ}$ have a considerably high value for the second cylinder, but they decrease for the third cylinder.

The presence of the two cylinders in front of the third one gives rise to a complex flow field. The acrosswind spectra of the third cylinder shows an increase in magnitude at low frequencies, but the shedding level remains the same. The low frequencies are primarily resulting from the increase in the lateral turbulence level. The alongwind spectra of the third cylinder shows an even broader and reduced peak for small angles. Figures for this configuration were not included for the sake of brevity.

\section{Configuration 3: Three Cylinders}

The lift force coefficient of the measurement cylinder in Configuration 3 is equal to zero due to the symmetry; however, the minimum drag coefficient occurs along T/D $=2$. For T/D $>2$, the drag force coefficient of the cylinder shows a minor change compared to the isolated cylinder. The total drag is greater as the spacing

ratio increased because each cylinder tends to behave as an isolated one ${ }^{13}$.

The generalized RMS drag coefficient and lift coefficient exhibit little variance when the test cylinder is 
upstream of the dummy cylinders, but significant changes appear when it is positioned downstream. The RMS drag coefficient shows peak at $\mathrm{L} / \mathrm{D}=3$ and 4 for $\mathrm{T} / \mathrm{D}=2$ and 3 , respectively. For $\mathrm{T} / \mathrm{D}=4$, the RMS drag shows large value further downstream. The RMS lift coefficients show the same trend as those of the RMS drag; however, the former exhibit considerably higher values than the latter.

It is interesting to note that the alongwind spectra in the case when the measurement cylinder is located downstream of the two dummy cylinders shows a very high peak at the shedding frequency and a second peak, albeit decreased in magnitude, at the double shedding frequency (see Fig. 3f). The energy level of the harmonic peak at T/D > 2 and $L / D>2$ is even higher than that of the acrosswind spectra. This feature indicates that very well organized vortices from the two upstream cylinders buffet the downstream cylinder, resulting in a peak in the spectrum at their frequency. The spectra for the cylinder when positioned upstream does not show any significant differences in behavior from the isolated cylinder.

When the spacing is decreased, the acrosswind spectra for the measurement cylinder at the upstream position show a suppressed peak, while the spectra for the cylinder at the downstream $L / D=2$ and 3 and T/D $=2$ and 3 (see Fig. 3e) indicate that the peak at the shedding frequency disappears, with two peaks, one at half the shedding frequency and the other at twice the shedding frequency, appearing. Both involve a somewhat very complex flow pattern that nevertheless exhibits a quasi-order nature. Further downstream, the spectra contain a peak at the shedding frequency with amplification of the energy level both at lower and higher frequencies in comparison to the isolated cylinder.

\section{Conclusions}

\section{Two Cylinder Case:}

This study presents the influence of interference due to proximity effects in a boundary layer flow for aerodynamic quantities such as mean and RMS lift and drag and their spectral descriptions. The mean and drag coefficients are in general agreement with previous studies. Large values of generalized RMS lift and drag coefficient occur when the measurement cylinder is buffeted by the wake of the upstream cylinder. A maximum value occurs between $\mathrm{S} / \mathrm{D}=3$ and 4 .

The spectra reveal that there is vortex formation and these vortices do impinge on the measurement cylinder. For smaller separations, such as S/D $<3$, the vortex shedding of both the upstream and downstream cylinders has been suppressed; however, when the downstream cylinder is positioned at a spacing ratio greater than three, the vortex shedding of the downstream cylinder is enhanced, whereas the upstream cylinder is hardly affected. Due to the vortex impingement, the downstream cylinder is subjected to the alongwind fluctuations at the shedding frequency and twice the shedding frequency.

\section{Three Cylinder Cases:}

Effects similar to those found in the two cylinder case were found for the second cylinder in an in-line arrangement of three cylinders. There is small difference between the second cylinder and third cylinder, only the third cylinder is affected more by low frequency fluctuations. When the downstream cylinder is exposed to dual wakes of the two side by side upstream cylinders, it is subjected to a large alongwind fluctuation which is comparable with the acrosswind fluctuation at the shedding frequency. For small separations, S/D $<3$, the spectra of the downstream cylinder exhibit double peaks at half the shedding frequency and twice the shedding frequency. 


\section{Acknowledgments}

The support for this work was provided in part by NSF Grant CMS94-02196 and CMS95-03779 and REU supplements.

\section{References}

[1] M.M. Zdrovkovich, Smoke observations of wakes of tandem cylinders at low Reynolds numbers, The Aeronautical Journal, Vol. 76, Feb. 1972, pp. 108-114.

[2] P.W. Bearman and A.J. Wadcock, The interaction between a pair of cylinders normal to a stream, J. Fluid Mechanics, Vol. 61 (1973) pp. 499-511.

[3] M.M. Zdravkovich and D.L. Pridden, Interference between two circular cylinders: series of unexpected discontinuities, J. Industrial Aerodynamics, 2(1977) 255-270.

[4] M.M. Zdravkovich, Review of flow interference between two circular cylinders in various arrangements, SME J. of Fluids Engineering, Vol. 99, No. 4, 1977, pp. 618-633.

[5] M. Arie, M. Kiya, M. Moriya and H. Mori, Pressure fluctuations on the surface of two circular cylinders in tandem arrangement, ASME, J. of Fluid Engineering, Vol. 105, 1983, pp. 161-167.

[6] C. Dalton and J.M. Szabo, Drag on a group of cylinders, ASME, J. of Pressure Vessel Technology, Feb. 1977, pp. $152-157$.

[7] M. Kiya, M. Arie, H. Tamura, and H. Mori, Vortex shedding from two circular cylinders in staggered arrangement, ASME, J. of Fluids Engineering, Vol. 102, 1980, pp. 166-173.

[8] A. Bokaian, F. Geoola, Wake induced galloping of two interfering circular cylinders, J. Fluids Mechanics, 1984, Vol. 146, pp. 383-415.

[9] S.C. Luo, et al., Uniform flow past one (or two in tandem) finite length circular cylinder(s), J of Wind Engineering and Industrial Aerodynamics, 59, 1996, pp. 69-93.

[10] T.F. Sun and Z.F. Gu, Interference between wind loading on group of structures, J of Wind Engineering and Industrial Aerodynamics, 54/55, 1995, pp. 213-225.

[11] T.F. Sun, Z.F. Gu, D.X. He, and L.L. Zhang, Fluctuating pressure on two circular cylinders at high reynolds numbers, J of Wind Engineering and Industrial Aerodynamics, 41-44, 1992, pp. 577-588.

[12] A. Kareem, C.M. Cheng, and Po Chien Lu, Aerodynamic pressure and force fluctuations on smooth cylinders, J. of Fluids and Structures, Vol. 3, 1989, pp. 481-508.

[13] A.T. Sayers, Flow interference between three equispaced cylinders when subjected to a cross flow, J of Wind 
Engineering and Industrial Aerodynamics, 26, 1987, pp. 1-19.

[14] Y. Ohya, A. Okajima, and M. Hayashi, Wake Interference and Vortex Shedding, Encyclopedia of Fluid Mechanics, Vol.8, Ch. 10, 1986, pp. 324-389.

[15] S. Taniguchi, H. Sakamoto and M. Aire, Interference between two circular cylinders of finite height vertically immersed in a turbulent boundary layer, ASME, J. of Fluids Engineering, Vol. 104, 1982, pp. 529-536.

[16] T.F. Sun and Z.F. Gu, Interference Between Wind Loading on Group of Structures, J Wind Engineering and Industrial Aerodynamics, 54-55, 1995, 213-225.

[17] S. Ishigai, E. Nishikawa, K. Nishimura, and K. Cho, Experimental Study of Structure of Gas Flow in Tube Banks With Tube Axes Normal to Flow (Part 1, Karman Vortex Flow around two Tubes at Various Spacings), JSME, 15, 86, 1972, pp. 949-956.

[18] M.M. Zdravkovich, Review of interference-induced oscillations in flow past 2 parallel circular cylinders in various arrangements, J of Wind Engineering and Industrial Aerodynamics, 28, 1988, pp. 183-200.

[19] W. Zhang and W.H. Melbourne, Interference between two circular cylinders in tandem in turbulent flow, J of Wind Engineering and Industrial Aerodynamics, 41-44, 1992, pp. 589-600.

[20] A. Okajima, K. Sugitani, and T. Mizota, Flow around a pair of circular cylinders arranged side by side at high Reynolds numbers, Trans. JSME, 52, 480, 1986, pp. 2844-2850.

[21] S.J. Price and M.P. Paidoussis, The aerodynamic forces acting in groups of two and three circular cylinders when subject to a cross-flow, J of Wind Engineering and Industrial Aerodynamics, 17, 1984, pp. 329-347.

[22] Y. Tanida, A. Okajima, and Y. Watanabe, Stability of a circular cylinder oscillating in uniform flow or in a wake, J. Fluid Mechanics, Vol. 3, 1989, pp. 481-508.

[23] M.E. Davies, A comparison of the wake structure of a stationary and oscillatory bluff body, using a conditional averaging technique, J. Fluid Mechanics, 1976, Vol. 75, pp. 209-231. 


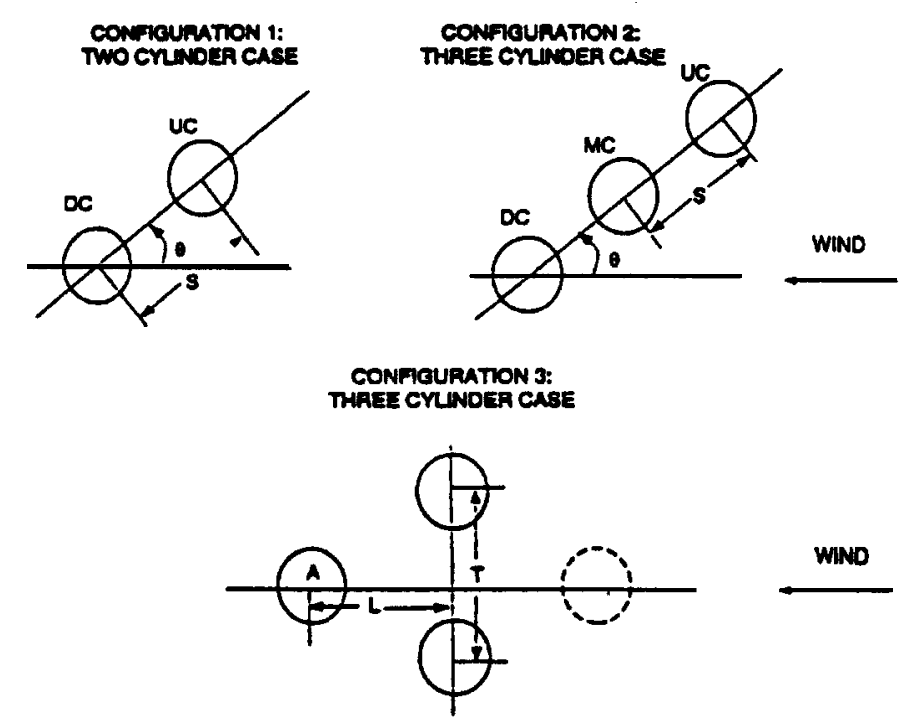

Fig. 1: Schematic of three cylinder configurations tested.

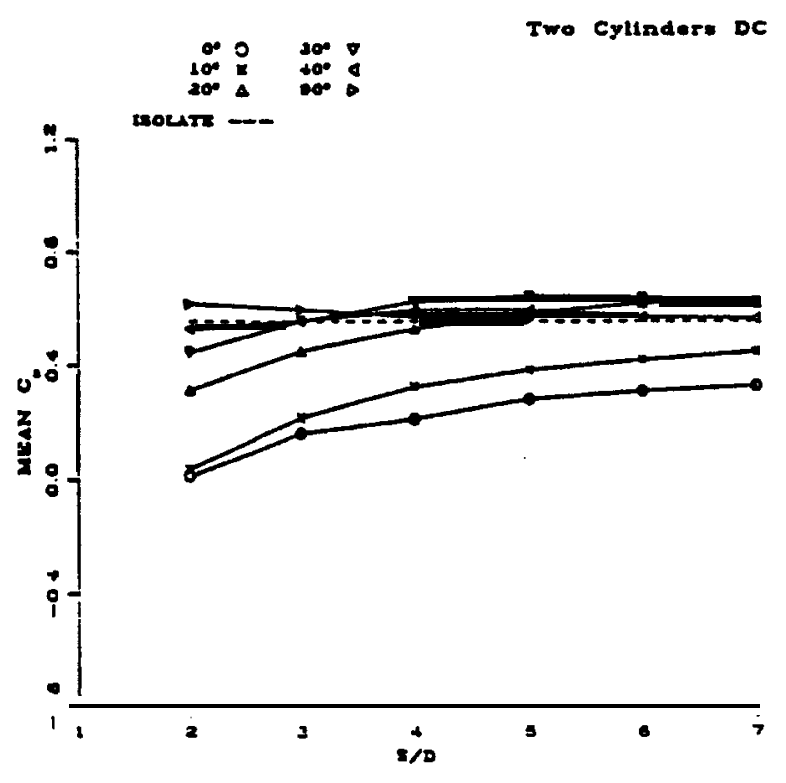

(a)

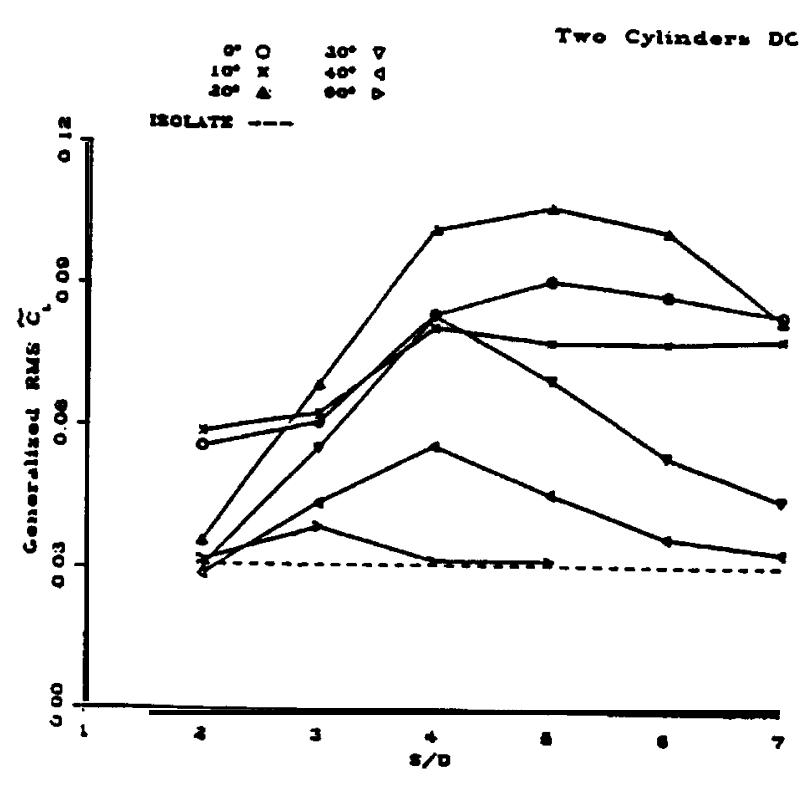

(b)

Fig. 2: (a) Mean drag force coefficient on downstream cylinder - configuration 1;

(b) RMS lift force coefficient on downstream cylinder - configuration 1. 


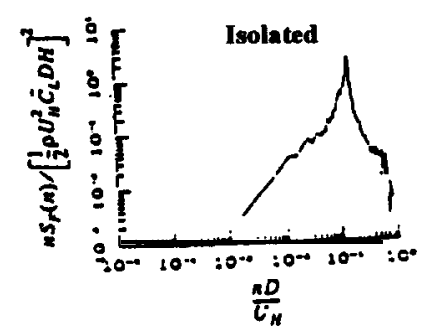

(a)

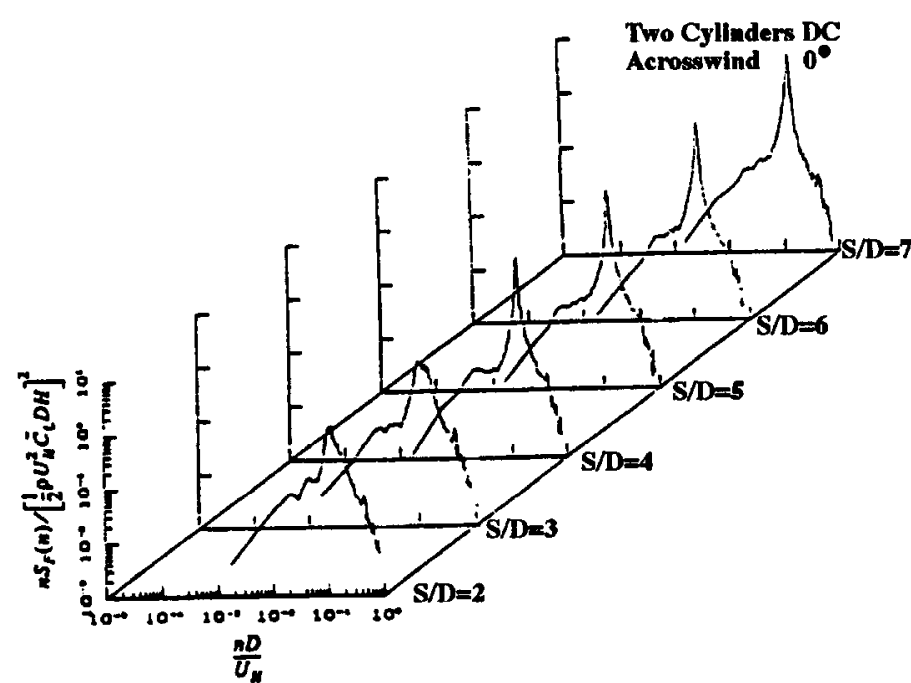

(c)

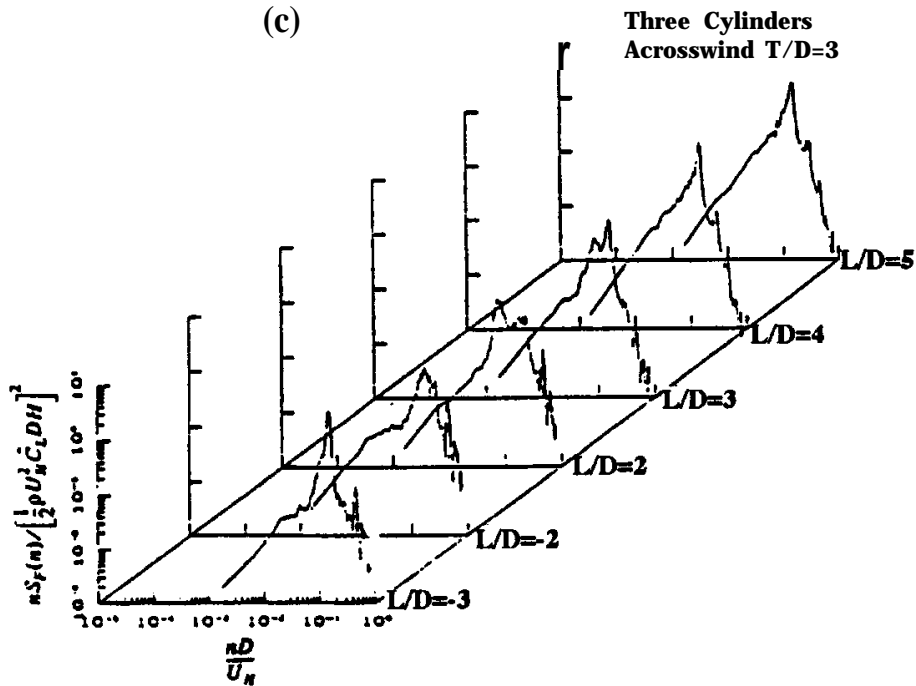

(c)

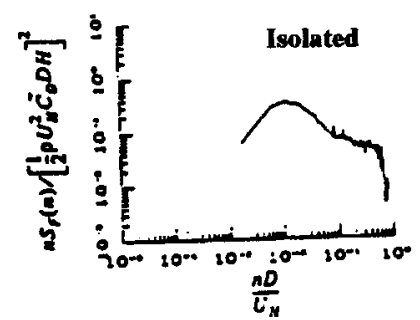

(b)

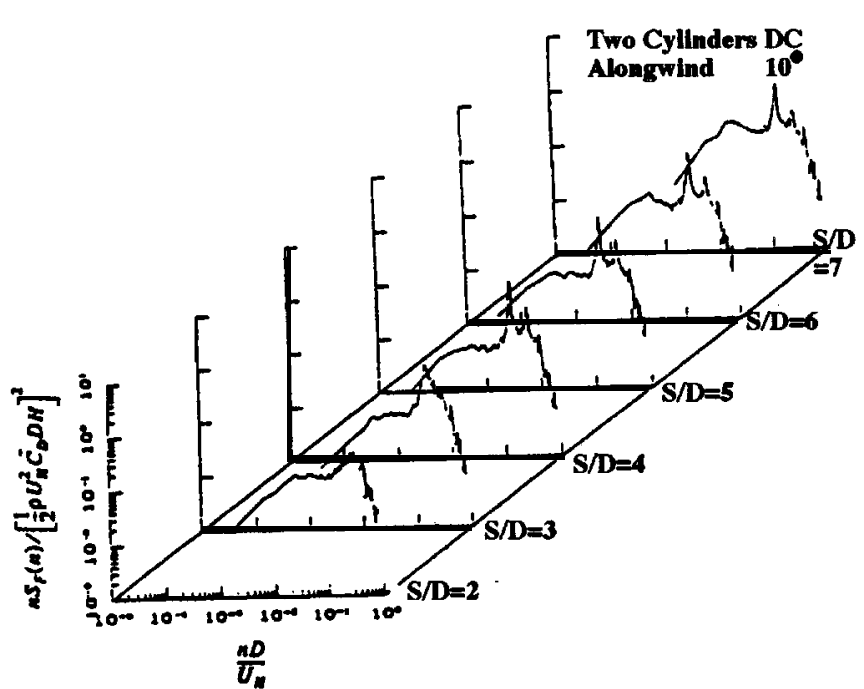

(d)

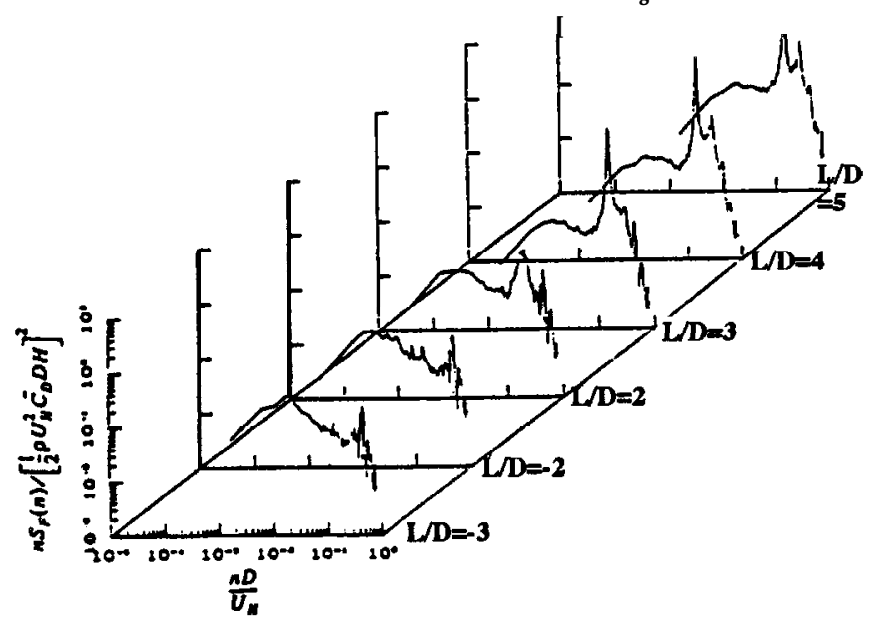

(f)

Fig. 3: (a) Spectra of RMS lift coefficient on isolated cylinder;

(b) Spectra of RMS drag coefficient on isolated cylinder;

(c) Spectra of RMS lift coefficient on downstream cylinder - configuration 1;

(d) Spectra of RMS drag coefficient on downstream cylinder - configuration 1;

(e) Spectra of RMS lift coefficient - configuration 3;

(f) Spectra of RMS drag coefficient - configuration 3. 